\title{
Characterization of glutathione transferases involved in the pathogenicity of Alternaria brassicicola
}

Benoit Calmes ${ }^{1,2,3}$, Mélanie Morel-Rouhier ${ }^{4,5}$, Nelly Bataillé-Simoneau ${ }^{1,2,3}$, Eric Gelhaye ${ }^{4,5}$, Thomas Guillemette ${ }^{1,2,3}$ and Philippe Simoneau ${ }^{1,2,3^{*}}$

\begin{abstract}
Background: Glutathione transferases (GSTs) represent an extended family of multifunctional proteins involved in detoxification processes and tolerance to oxidative stress. We thus anticipated that some GSTs could play an essential role in the protection of fungal necrotrophs against plant-derived toxic metabolites and reactive oxygen species that accumulate at the host-pathogen interface during infection.

Results: Mining the genome of the necrotrophic Brassica pathogen Alternaria brassicicola for glutathione transferase revealed 23 sequences, 17 of which could be clustered into the main classes previously defined for fungal GSTs and six were 'orphans'. Five isothiocyanate-inducible GSTs from five different classes were more thoroughly investigated. Analysis of their catalytic properties revealed that two GSTs, belonging to the GSTFuA and GTT1 classes, exhibited GSH transferase activity with isothiocyanates (ITC) and peroxidase activity with cumene hydroperoxide, respectively. Mutant deficient for these two GSTs were however neither more susceptible to ITC nor less aggressive than the wild-type parental strain. By contrast mutants deficient for two other GSTs, belonging to the Ure2pB and GSTO classes, were distinguished by their hyper-susceptibility to ITC and low aggressiveness against Brassica oleracea. In particular AbGSTO1 could participate in cell tolerance to ITC due to its glutathione-dependent thioltransferase activity. The fifth ITC-inducible GST belonged to the MAPEG class and although it was not possible to produce the soluble active form of this protein in a bacterial expression system, the corresponding deficient mutant failed to develop normal symptoms on host plant tissues.
\end{abstract}

Conclusions: Among the five ITC-inducible GSTs analyzed in this study, three were found essential for full aggressiveness of $A$. brassicicola on host plant. This, to our knowledge is the first evidence that GSTs might be essential virulence factors for fungal necrotrophs.

Keywords: Glutathione transferases, Necrotrophic fungi, Pathogenesis

\section{Background}

Alternaria brassicicola is the causative agent of black spot disease in a wide range of Brassicaceae crops. The necrotrophic behavior of this fungus exposes it to several plant defense compounds such as phytoanticipins and phytoalexins $[1,2]$ during host colonization. Brassicaceae phytoanticipins are represented by glucosinolates that are hydrolyzed by myrosinase when the plant tissues are

\footnotetext{
* Correspondence: philippe.simoneau@univ-angers.fr

'Université d'Angers, UMR 1345 IRHS, SFR 4207 QUASAV, 2 Bd Lavoisier, Angers cedex F-49045, France

${ }^{2}$ INRA, UMR 1345 IRHS, 42 rue Georges Morel, Beaucouzé Cedex F-49071, France Full list of author information is available at the end of the article
}

damaged during necrotrophic colonization. Brassicaceae contain a large variety of glucosinolates, each of which form different isothiocyanates (ITCs) when hydrolyzed such as allyl-ITC (Al-ITC), benzyl-ITC (Bz-ITC) or phenylethyl-ITC (Ph-ITC) [3]. ITCs, which are the major breakdown compounds of glucosinolates [4], have been shown to exert their toxicity towards $A$. brassicicola by oxidative stress generation [5].

Glutathione transferases (GSTs) are a superfamily of proteins which are found widespread in animals, plants, fungi and bacteria. GSTs usually catalyze glutathione (GSH) transfer onto hydrophobic molecules (glutathionylation

\section{Biomed Central}


activity), or GSH removal from specific substrates (deglutathionylation) [6]. The varieties of electrophilic compounds, which can be conjugated to GSH by GSTs include aliphatic and aromatic halogen compounds, peroxides and epoxides, $\alpha, \beta$-unsaturated and low molecular weight proteins. In particular, it has been shown that GSTs are able to conjugate GSH to ITC in human, Arabidopsis thaliana and Phanerochaete chrysosporium [7-9]. In the same vein, GSTs play a major role in insect adaptation to plant secondary compounds, such as glucosinolates (GLS) and ITCs in the polyphagous aphid species Myzus persicae [10]. In addition, ITCs specifically induce GSTs in Caenorhabditis elegans, providing oxidative stress tolerance [11]. Concerning fungal pathosystems, induction of GST encoding genes and increased transferase activity were reported in Sclerotinia sclerotiorum during Brassica napus infection and in the presence of ITC [12]. Moreover, some GSTs have also been identified as ligandin proteins that selectively bind organic anions such as tetrapyrroles in mammals and plants $[13,14]$. This ligandin property has been defined as the capacity of the protein to bind non-substrate ligands [13]. In plants, it could be involved in intracellular transport of hydrophobic compounds such as pigments, and in temporary storage of phytohormones $[15,16]$. In fungi, this ligandin property has been described for members of the GSTFuA class in P. chrysosporium [17]. These enzymes are able to bind wood-derived molecules and could participate in the intracellular transport and further elimination of these compounds, which could be toxic for the cells. Several GSTs also play a direct role in the antioxidant response through their peroxidase activity, which reduces endogenous or exogenous hydrogen peroxides or fatty acid peroxides $[18,19]$. GSTs can usually accept various substrates. This functional property allows them to detoxify a wide range of endogenous and environmental chemicals and is part of their evolution in response to selective pressure. In Saccharomyces pombe and Aspergillus fumigatus, GSTs are involved in the oxidative stress response. Their gene expression is induced by hydrogen peroxide $\left(\mathrm{H}_{2} \mathrm{O}_{2}\right)$. The promoters contain multiple copies of the stress response element (STRE) consensus region and the binding site of the Yap1 transcription factor, known to modulate the adaptive response to oxidative stress or cytotoxic agents [20]. In yeast, the GTT1 gene promoter contains specific regions of the response to xenobiotics [21] but GST regulation is usually the result of post-transcriptional modifications [22].

The pathogenicity of A. brassicicola could be partly related to its ability to protect itself against Brassicaceae defenses compounds including ITC [23-25]. The results obtained by $[5,26]$ showed that at least six genes encoding GST in A. brassicicola, were up-regulated upon exposure to ITC. One of these GSTs, named AbGST1, which is also up-regulated during interaction with the host plant has been the focus of a more detailed study
[26]. AbGST1 transcription was found to be significantly enhanced by heavy metals and 1-chloro-2,4-dinitrobenzene $(\mathrm{CDNB})$ and the recombinant protein exhibited high glutathione transferase activity with allyl and benzyl ITC as substrate as compared to CDNB. In the present study, we functionally characterized the other five ITCinducible GSTs as well as the phenotype of mutants deficient for these enzymes. Our results indicate that three of these GSTs, belonging to the GSTO, Ure2pB and MAPEG classes, may contribute to pathogenicity probably by protecting the fungus against the oxidative stress generated by host plant defense compounds.

\section{Results}

Phylogenetic analysis of GST- coding sequences from $A$. brassicicola

Searching the A. brassicicola genome (http://genome.jgipsf.org/Altbr1/Altbr1.home.html) with glutathione transferase as keyword generated 25 entries. Sequence analyses revealed that 23 of them might correspond to true GSTs. A phylogenetic analysis focusing on GSTs from 8 Ascomycetes species and 1 Basidiomycete fungus allowed the clustering of most sequences into the main previously defined classes [27-29] (Fig. 1). A. brassicicola clearly exhibited 3 Ure2pB sequences but no Ure2pA, 1 EFB, 5 GTT1, 3 GHR, 2 GSTO, 2 GSTFuA and 1 MAPEG sequences. However, attribution of the other six sequences to previously identified groups was not clear. In particular, two sequences clustered close to P. chrysosporium GTT2, but their sequences contained domains related to beta or sigma GSTs that are usually found in bacteria and humans, respectively. Moreover, they were not related to the yeast GTT2 isoform. These sequences have thus not yet been classified. No member of the newly described phi class [30] was found.

In a previous study, five of these GST coding genes have been found up-regulated upon exposure to ITC [5]. Based on the present phylogenetic analysis, they were renamed as follows: AbGTT1.2 (AB05243.1), AbGSTO1 (AB04746.1), AbMAPEG1 (AB08663.1), AbUre2pB1 (AB09895.1) and AbGSTFuA1 (AB07364.1) corresponding to ITC-induced ESTs A1F1, A2C1, A2H5, A2C10 and A4D12, respectively [5] (Table 1). Similarly, the previously characterized AbGST1 from A. brassicicola [26] was renamed AbGTT1.1 (AB05341.1).

\section{Enzymatic activities of selected AbGST proteins}

Recombinant AbGTT1.2, AbUre2pB1, AbGSTFuA1 and AbGSTO1 proteins were produced in Escherichia coli and purified. Despite several attempts to optimize the production parameters, AbMAPEG1 was always found in the insoluble fraction and could thus not be purified in its active form. The enzymatic activities of the recombinant proteins were determined using $\mathrm{CDNB}$ as a classical substrate 


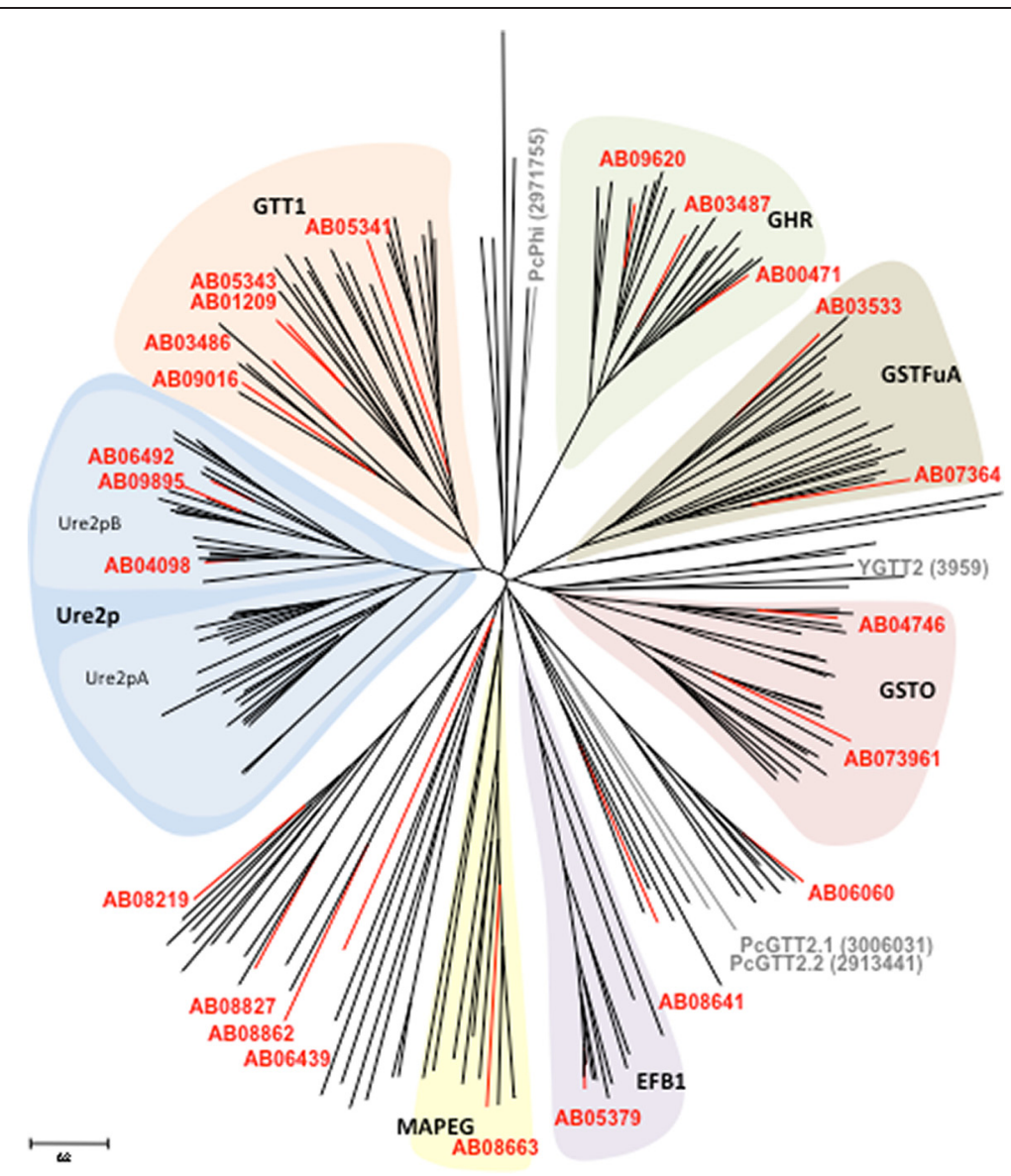

Fig. 1 Phylogenetic tree of ascomycete glutathione transferases. Sequences from the basidiomycete Phanerochaete chrysosporium were added to allow GST classification into the following classes: Ure2p, GSTFuA, Omega (GSTO), Glutathionyl Hydroquinone Reductase (GHR), EFBY, GTT1 and MAPEG. The sequences (Additional file 3) were retrieved from genomes of Saccharomyces cerevisiae, Phanerochaete chrysosporium (PC), Alternaria brassicicola, Botrytis cinerea, Leptosphaeria maculans, Mycosphaerella figensis, Mycosphaerella graminicola, Neurospora crassa and Stagonospora nodorum available at the Joint Genome Institute (http://www.jgi.doe.gov/). Sequence alignments were done by CLUSTALW and the tree was constructed with the Neighbor joining method. The scale marker represents 0.2 substitutions per residue. Data available from the Dryad Digital Repository: http://dx.doi.org/10.5061/dryad.19f18

for glutathione transferase assay. Various ITCs were also used (Al-ITC, Bz-ITC and Ph-ITC) and peroxidase activities were tested using $\mathrm{H}_{2} \mathrm{O}_{2}$ and cumene hydroperoxide $(\mathrm{Cu}-\mathrm{OOH})$ (Table 2). Finally deglutathionylation activity was assayed using $\beta$-ME-SG. AbGTT1.2 and AbUre2pB1 exhibited both GSH transferase with CDNB and peroxidase activity with $\mathrm{Cu}-\mathrm{OOH}$. No activity was detected with $\mathrm{H}_{2} \mathrm{O}_{2}$. AbGSTFuA1 was found to be the most

Table 1 Correspondence between EST, protein accession numbers and AbGST names derived from the phylogenetic analysis

\begin{tabular}{lll}
\hline EST & Protein & GST name \\
\hline A1F1 & AB05243.1 & AbGT1.2 \\
A2C1 & AB04746.1 & AbGSTO1 \\
A2H5 & AB08663.1 & AbMAPEG1 \\
A2C10 & AB09895.1 & AbUre2pB1 \\
A4D12 & AB07364.1 & AbGSTFuA1 \\
\hline
\end{tabular}

active protein in our tests, exhibiting significant GSH transferase activities with all ITCs. AbGSTO1 exhibited a deglutathionylating activity with $\beta$-ME-SG. These enzymatic profiles are in accordance with those identified within each class for other fungal species except for Ure2pB1 [9, 27, 29, 31, 32]. The Ure2p class was split into two subclasses (Ure2pA and Ure2pB). AbUre2pB1 clustered within the Ure2pB subclass, however it exhibited a glutathionylation activity like the yeast isoform, which belongs to the Ure2pA subclass, rather than the deglutathionylation activity previously measured for Ure $2 \mathrm{pB}$ in $P$. chrysposporium and E. coli [33, 34].

\section{Gene expression of GSTs during plant infection and in response to oxidative stress}

Gene expression of all the selected GSTs was previously shown to be ITC-inducible. Their expression was therefore checked during in planta interaction with Brassica 
Table 2 Kinetic parameters of AbGSTs in enzymatic assays

\begin{tabular}{lllll}
\hline & AbGTT1.2 & AbGSTO1 & AbUre2pB1 & AbGSTFuA1 \\
\hline $\boldsymbol{K}_{\mathbf{m}}(\mathbf{m M})$ & & & & \\
CDNB & $1.82 \pm 0.33$ & $\mathrm{ND}$ & $5.01 \pm 0.40$ & $0.40 \pm 0.02$ \\
Al-ITC & $\mathrm{ND}$ & $\mathrm{ND}$ & $\mathrm{ND}$ & $0.12 \pm 0.02$ \\
Bz-ITC & $\mathrm{ND}$ & $\mathrm{ND}$ & $\mathrm{ND}$ & $0.18 \pm 0.03$ \\
Ph-ITC & $\mathrm{ND}$ & $\mathrm{ND}$ & $\mathrm{ND}$ & $0.11 \pm 0.02$ \\
$\mathrm{Cu}-\mathrm{OOH}$ & $0.12 \pm 0.04$ & $\mathrm{ND}$ & $0.77 \pm 0.06$ & $\mathrm{ND}$ \\
$\beta-\mathrm{ME}-\mathrm{SG}$ & $\mathrm{ND}$ & $1.12 \pm 0.02$ & $\mathrm{ND}$ & $\mathrm{ND}$ \\
GSH & $1.33 \pm 0.10$ & $0.45 \pm 0.13$ & $0.87 \pm 0.02$ & $0.50 \pm 0.0004$ \\
$\boldsymbol{k}_{\text {cat }}\left(\mathbf{s}^{-1}\right)$ & & & & \\
CDNB & $0.10 \pm 0.05$ & $\mathrm{ND}$ & $1.50 \pm 0.08$ & $38.18 \pm 6.10$ \\
Al-ITC & $\mathrm{ND}$ & $\mathrm{ND}$ & $\mathrm{ND}$ & $76.80 \pm 11.64$ \\
Bz-ITC & $\mathrm{ND}$ & $\mathrm{ND}$ & $\mathrm{ND}$ & $11.81 \pm 1.82$ \\
Ph-ITC & $\mathrm{ND}$ & $\mathrm{ND}$ & $\mathrm{ND}$ & $20.54 \pm 2.96$ \\
Cu-OOH & $6.82 \pm 1.18$ & $\mathrm{ND}$ & $0.20 \pm 0.01$ & $\mathrm{ND}$ \\
$\beta$-ME-SG & $\mathrm{ND}$ & $49.00 \pm 9.40$ & $\mathrm{ND}$ & $\mathrm{ND}$ \\
\hline
\end{tabular}

The apparent $K_{\mathrm{m}}$ values for all compounds were determined using a 0.1-50 mM concentration range in the presence of $3 \mathrm{mM} \mathrm{GSH}$. The $K_{\mathrm{m}}$ value for GSH was determined with $2 \mathrm{mM}$ CDNB and a 0.01 to $10 \mathrm{mM} \mathrm{GSH}$ concentration range. The apparent $K_{\mathrm{m}}$ and $k_{\text {cat }}$ values were calculated by nonlinear regression using the Michaelis-Menten equation $(r 2>0.99)$. Data are represented as mean \pm S.D. $(n \pm 3)$

ND Not detectable

oleracea (Fig. 2a). Genes encoding the five GSTs were up-regulated compared to the control (in vitro development conditions). AbGTT1.2 gene was most strongly and quickly ( 2 dpi, i.e. when the necrotic area around the inoculation point was barely visible) induced during the interaction. The maximum expression level was reached at 6 dpi (i.e. extending necrosis with fungal conidia formed on the surface of infected tissues) for most of the GSTs. Peak of expression was particularly marked for the genes encoding AbGTT1.2 and AbGSTO1. Overall, the transcription induction of the five selected genes occurred very rapidly during infection and remained high throughout the interaction. As ITCs have been found to generate oxidative stress in fungi [5], expression of the five GST genes was also recorded in fungal cultures supplemented with $\mathrm{H}_{2} \mathrm{O}_{2}$ (Fig. 2b). Strong upregulation of all the selected $A b G S T$ genes was observed after $30 \mathrm{~min}$ exposure and then the transcripts returned to their basal level after $2 \mathrm{~h}$.

\section{Pathogenic behavior of GST-deficient mutants in planta}

Virulence and aggressiveness of the GST-deficient mutants were evaluated on $B$. oleracea. Six days after inoculation of a highly concentrated conidia suspension $\left(10^{5}\right.$ conidia per ml), usual symptoms were observed for the wild-type and mutant genotypes (data not shown). At high parasitic pressure, the loss of one of the fiveselected AbGSTs did not affect the virulence of $A$. brassicicola. The analysis of symptoms under lower applied parasitic pressure $\left(10^{4}\right.$ conidia per $\left.\mathrm{ml}\right)$ revealed differences in aggressiveness between the tested genotypes (Fig. 3). $\triangle A b G T T 1.2$ and $\triangle A b G S T F u A 1$ mutants showed no significant difference compared to the wild-type when the inoculum was at low concentration. For $\triangle A b G S T O 1$, $\triangle A b M A P E G 1$ and $\triangle A b U r e 2 p B 1$ a significant decrease in aggressiveness compared to the wild-type strain was observed at $6 \mathrm{dpi}$. The necrotic area decreased by approximately $70 \%$ for these mutants.

\section{Susceptibility of GST-deficient mutants to isothiocyanates} The effects of $A b G S T$ inactivation in A. brassicicola on conidia germination and initial mycelium growth in the presence of ITCs were examined. An analysis of the nephelometric growth curves (Fig. 4a) revealed that under control condition (PDB medium), no significant phenotypic difference in conidia germination (based on the lag time parameter) or mycelium growth (based on the maximum growth rate parameter) was detected in any of the tested mutants as compared to the wild-type. In contrast, mutants deficient for AbGSTO1 and for AbUre2pB1 showed
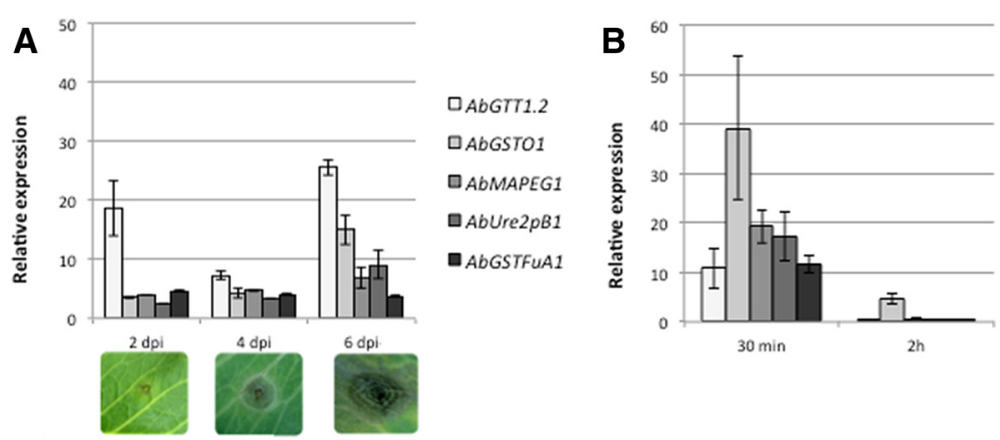

Fig. 2 Quantitative RT-PCR results for the expression of selected GST genes. Gene expression was measured either in planta at 2, 4 and 6 day post inoculation (dpi) (a) or in vitro in the presence of $\mathrm{H}_{2} \mathrm{O}_{2}(2.5 \mathrm{mM})$ for 30 min and $2 \mathrm{~h}$ (b). For each gene, expression induction is represented as a ratio of its relative expression (studied gene transcript abundance/actin and tubulin transcript abundance) in each inductive condition to its relative expression in the corresponding control. The data are the mean of three repetitions 


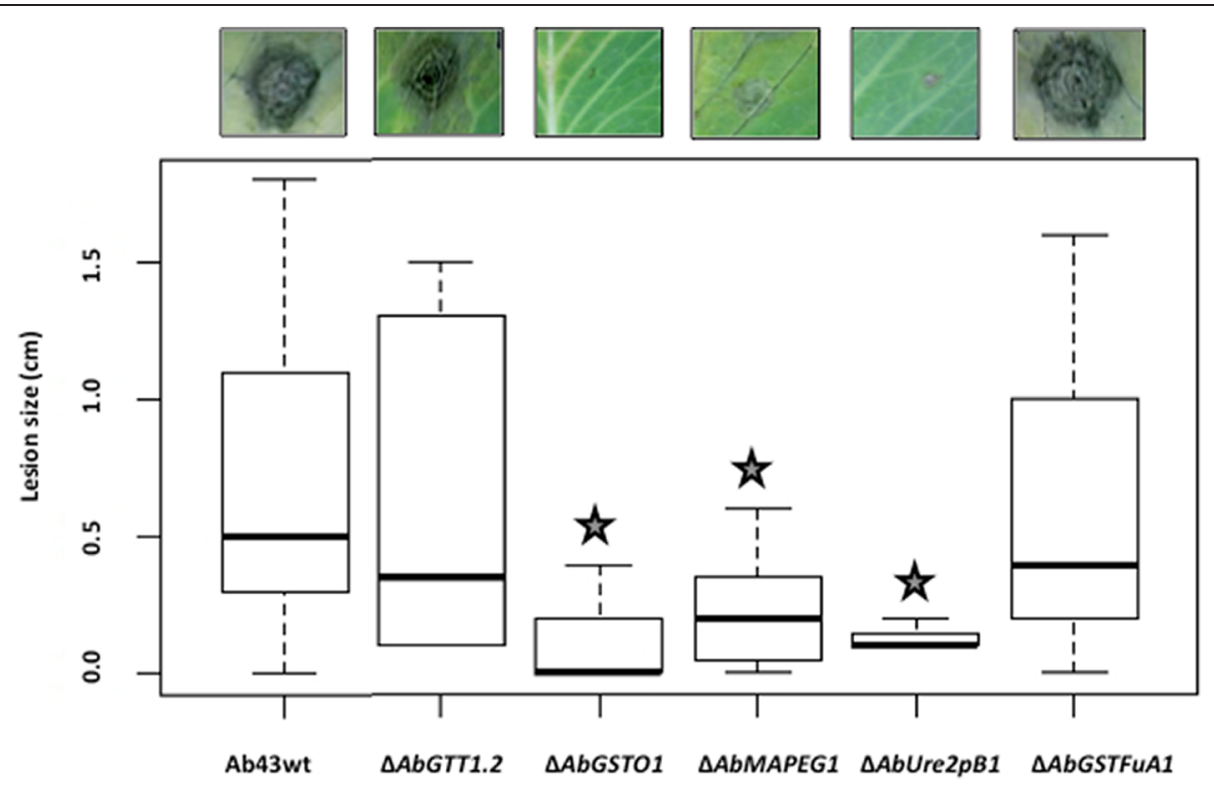

Fig. 3 Pathogenic behaviour of GST-deficient mutants. B. oleracea leaves were inoculated with $5 \mu \mathrm{L}$ drops of conidia suspension $\left(10^{4} \mathrm{conidia} / \mathrm{mL}\right.$ in water). Transformants were inoculated on the right part of the central vein and compared on the same leaf with the parental strain (inoculated on the left part of the central vein). Percentages of aggressiveness with respect to the wild-type strain were calculated at 6 dpi. Stars indicate a significant difference with respect to the wild-type aggressiveness $(100 \%)$ using the Student test $(P<0.01)$

an increased susceptibility to ITC compared to the wild type (Fig. 4b). In comparison, $\triangle A b G S T F u A 1$ behaved like the wild-type strain and $\triangle A b G T T 1.2, \triangle A b M A P E G 1 \mathrm{had}$ an intermediate phenotype.

\section{Discussion}

In A. brassicicola, the first characterized GST, initially called AbGST1 [26] and renamed AbGTT1.1 in the present study as it belongs to the GTT1 family, was shown to possess conjugation activity of Al-ITC or CDNB to glutathione. A more thorough examination of the A. brassicicola proteome revealed 22 more putative GSTs that could be classified into 7 families according to the proposed classification. Investigation of the GST content in several fungal genomes revealed a huge diversity in terms of GST number ranging from 6 in S. cerevisiae to more than 40 in saprophytic Basidiomycetes [30]. However, the number of GSTs encoded by the A. brassicicola genome was comparable to that found in other fungal necrotrophs such as Botrytis cinerea (24), Stagonospora nodorum (23), or hemibiotrophs such as Mycosphaerella graminicola (25), Mycosphaerella fijensis (28), Leptosphaeria maculans (23). Five of the A. brassicicola GSTs that were previously shown to be up-regulated during ITC exposure [5] were clustered into five different classes, i.e. GTT1, GST Omega, GSTFuA, Ure2p and MAPEG.

Detailed analysis of the catalytic properties of these ITC-inducible GSTs revealed that, besides AbGTT1.1, another GST, called AbGSTFuA1, was able to conjugate
ITC to glutathione. Members of the fungus-specific GSTFuA class have been described as having ligandin properties with various small aromatic compounds and GSH transferase activity with phenetyl-ITC [9]. This GST therefore appears to be a good candidate in protection mechanisms of $A$. brassicicola against host fungitoxic metabolites. However the mutant strain deficient for this enzyme was neither more susceptible to ITC nor less aggressive on Brassica than the wild-type and thus did not appear to be crucial for A. brassicicola pathogenicity. This could be due to a putative functional redundancy with the second isoform identified in the $A$. brassicicola genome. Otherwise it could be due to the fact that, in presence of high ITC concentrations, the efficient conjugation activity of AbGSTFuA1 could result in intracellular GSH depletion, leading to overall cellular oxidative stress. Indeed, Zhang [35] reported that ITCs are presumed to penetrate human and animal cells by diffusion but once inside the cells they rapidly conjugated via their $-\mathrm{N}=\mathrm{C}=\mathrm{S}$ group with intracellular $\mathrm{GSH}$. Such conjugation, which takes place spontaneously but is enhanced by GST, may explain the rapid accumulation of ITC within the cells (up to 100-fold the extracellular concentration) and the rapid and marked depletion of GSH observed after ITC exposure [36, 37].

AbGTT1.2 is a second member of the GTT1 class of ITC-inducible GST in A. brasiscicola. However, unlike AbGTT1.1, AbGTT1.2 was able to conjugate glutathione to CDNB but was not found to accept ITCs as substrate and had a highly efficient peroxidase activity against 

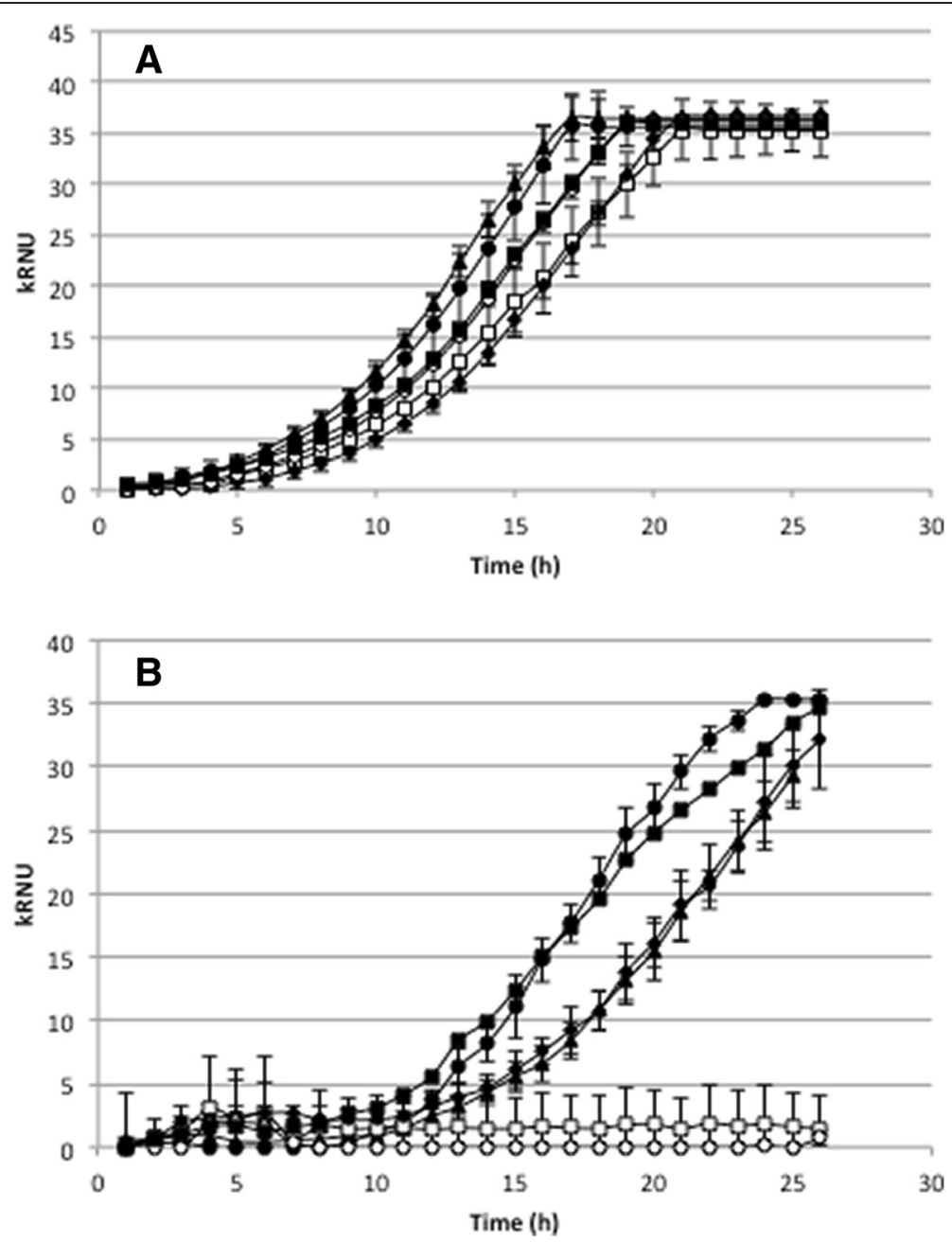

Fig. 4 Nephelometric monitoring of growth of the wild-type strain and AbGST deficient mutants. Conidia from the wild-type and mutants were used to inoculate microplate wells containing standard PDB medium (a) or supplemented with Ph-ITC (b). Growth was automatically recorded for $25 \mathrm{~h}$ at $25^{\circ} \mathrm{C}$ using a nephelometric reader. Each genotype was analyzed in triplicate and the experiments were repeated three times per growth condition. Black circles: wild-type strain; black triangles: $\triangle A b G T 1.2$; white squares: $\triangle A b G S T O 1$; black diamonds: $\triangle A b M A P E G 1$; white circles: $\triangle A b U$ re2pB1; black squares: $\triangle A B G S T F U A 1$

cumene hydroperoxide. In $S$. cerevisiae, GTT1 was shown to catalyse the reduction of hydroperoxides [38] and to be involved in xenobiotic detoxification [39]. Strong in planta AbGTT1.2 gene induction was observed during fungal infection, but the $\triangle A b G T T 1.2 \mathrm{mu}$ tant did not show reduced aggressiveness on its host plant or increased susceptibility to ITC compared to the wild-type. As five members of the GTT1 class were found in A. brassicicola, the absence of a marked phenotype for the AbGTT1.2-deficient mutant suggested that these GSTs have partial functional redundancies in this fungal pathogen.

By contrast with the two above-mentioned GST-deficient mutants, $\triangle A b M A P E G 1, \triangle A b U r e 2 p B 1$ and $\triangle A b G S T O 1$ mutants were highly impaired in their pathogenicity, with a severe reduction in their ability to form extended necrosis on host tissues. The two latter mutants were also found to be much more susceptible than the wildtype to ITC exposure. AbGSTO1 belongs to the Omega family, a member of the cysteine-containing GSTs superfamily that is widespread in several kingdoms and phyla [40]. The conserved cysteine residue in their active site modifies their enzymatic properties as they do not catalyze conventional conjugation reactions but instead have glutathione-dependent thioltransferase activity like many other cysteine-containing GSTs [41]. In line with its apparent protective role against ITC toxicity, AbGSTO1 protein may therefore be involved in the reduction of sulfur bonds formed after reaction between ITC and cysteine sulfhydryl groups of target cellular proteins, thus restoring their enzymatic functions. Indeed, in human cells, ITCs were found to covalently 
bind to multiple cysteine residues of different target proteins such as $\alpha$ and $\beta$-tubulin [42], Toll-like receptor TLR4 [43] or cytochrome P450 enzymes [44].

AbUre2pB1, as noted with AbGTT1.2, was found to function as GST and peroxidase although the measured activities were low. However, contrary to the $\triangle A b G T T 1.2$ mutant, the $\triangle A b U r e 2 p B 1$ mutant showed increased susceptibility to ITC and decreased aggressiveness compared to the wild-type strain. The first structural and biochemical characterization of a fungal Ure2pB GST (PcUre2pB1 from Phanerochaete chrysosporium) was recently published [33]. Unlike PcUre2pB1, AbUre2pB1 did not depict any activity in deglutathionylation tests with $\beta$-ME-SG. Based on our results, it is therefore difficult to come up with any convincing explanation concerning the role of this enzyme in protection against ITCs and in pathogenesis, but they raise interesting questions concerning the evolution of these enzymes. It should be noted that, besides small molecules, PcUre2pB1 was also shown to be involved in glutathionylation/deglutathionylation of proteins, particularly to interact with a GST from the omega class of P. chrysosporium, PcGSTO3 [33]. As AbGSTO1 and AbUre2pB1 have comparable expression patterns and mutants deficient for these two GSTs have similar phenotypes, it would be interesting to check whether these two proteins could also interact and cooperate to protect the fungal cell against ITC toxicity. We have not been able to produce the soluble active form of AbMAPEG1 in a bacterial expression system. In fact, active recombinant forms of eukaryotic members of these membrane-associated GSTs have mainly been obtained in yeast or baculovirus/insect expression systems [45, 46]. However, careful inspection of the protein sequences derived from AB08663.1 (corresponding to the A2H5 ITC-induced EST, [5]) revealed the presence of a sequence pattern similar to that described by Bresell et al. [45] for the MGST3 sub-family of microsomal glutathione transferase (MAPEG) and a typical MAPEG hydrophobicity profile (Additional file 1). This protein has been shown to exhibit peroxidase activity and the corresponding gene is up-regulated in P. chrysosporium in the presence of toxic oak extracts, suggesting a putative role in the oxidative stress response [47]. The phenotype of the mutant defective for AbMAPEG1 suggested that this protein had no function in ITC detoxication while being important for full pathogenicity. Examination of microarray data from A. brassicicola exposed to brassicaceous indolic phytolexins (N'Guyen, unpublished) revealed that among the 23 GSTs, 6 were up-regulated in the presence of brassinin but of these only AbMAPEG1 belongs to the ITC-inducible set considered in the present study. This suggests that AbMAPEG1 could be involved in brassinin tolerance during host-plant colonization.

\section{Conclusions}

Three of the ITC-inducible GSTs were found to be essential for the normal pathogenicity of A. brassicicola. So far functional characterization of fungal GSTs has mainly been performed with saprophytic model species such as $A$. nidulans and $S$. cerevisiae or wood-degrading species like $P$. chrysosporium. A mutant deficient for a GST $(\triangle B c G S T 1)$ was obtained in $B$. cinerea but did not show any reduced virulence [48]. To the best of our knowledge this study therefore provided the first evidence that some GSTs play an essential role in the pathogenicity of a fungal necrotroph.

\section{Methods}

\section{Fungal strains and growth conditions}

The A. brassicicola wild-type strain Abra43 used in this study has previously been described [23, 24]. For routine culture, A. brassicicola was grown and maintained on potato dextrose agar (PDA). The method based on microscale liquid cultivation (from conidial suspensions) and automated nephelometric recording of growth, followed by extraction of relevant variables (lag time and growth rate), was described by [49]. To study the susceptibility of fungal strains to ITCs, allylITC (Al-ITC), benzyl-ITC (Bz-ITC) or phenetyl-ITC (Ph-ITC), all purchased from Aldrich Chemical Co. (Milwaukee, WI), were diluted from stock solutions prepared in methanol at the final desired concentrations. Solvent concentrations in controls and assays did not exceed $1 \%(\mathrm{v} / \mathrm{v})$.

\section{RNA isolation and expression analysis by real-time quantitative PCR}

Total RNA was prepared according to the TRIzol reagent protocol (Invitrogen). Additional cleanup and DNase treatment were performed using the Nucleospin RNA II kit (Macherey-Nagel) according to the manufacturer's protocol. Complementary DNA was synthesized from $5 \mu \mathrm{g}$ of total RNA using the reverse-transcription system [50 mM Tris- $\mathrm{HCl} \mathrm{pH}$ 7.5, $75 \mathrm{mM} \mathrm{KCl,} 10 \mathrm{mM}$ DTT, $3 \mathrm{mM} \mathrm{MgCl} 2,400 \mathrm{nM}$ oligo(dT) ${ }_{15}, 1 \mathrm{mM}$ random hexamers, $0.5 \mathrm{mM}$ dNTP, 200 units M-MLV reverse transcriptase, Promega]. The total volume was adjusted to $30 \mu \mathrm{l}$ and the mixture was then incubated for $60 \mathrm{~min}$ at $42{ }^{\circ} \mathrm{C}$. Aliquots of the resulting first-strand cDNA were used for real-time PCR amplification experiments using the $A B I$ Prism 7000 sequence detection system (Applied Biosystems) and the SYBR green PCR master mix according to the manufacturer's instructions. After 10 min denaturation at $95{ }^{\circ} \mathrm{C}$, the reactions were cycled 40 times at $95{ }^{\circ} \mathrm{C}$ for $15 \mathrm{~s}$ and $60^{\circ} \mathrm{C}$ for $1 \mathrm{~min}$. The absence of contaminating genomic DNA in the RNA samples was checked by direct amplification of non-reverse transcribed samples. The 
synthesis of a single specific PCR product was verified by melting point analysis after the run. For each condition, all amplifications were performed in triplicate from two separate biological samples and the mean was determined for further calculations. The relative quantification analysis was performed using the comparative $\Delta \Delta \mathrm{Ct}$ method as described by [50]. To evaluate the gene expression level, the results were normalized using $\mathrm{Ct}$ values obtained from actin cDNA amplifications run on the same plate.

\section{Generation of targeted gene replacement constructs and fungal transformation}

The gene replacement cassettes were generated using the double-joint PCR procedure [51]. The selectable marker inserted in the PCR constructs corresponded to the $\mathrm{Hph}$ gene cassette (1436 bp) from pCB1636 [52] conferring resistance to hygromycin $B$. The sets of primers used to amplify the 5 ' and 3 ' flanking regions of each targeted gene are presented in the Additional file 2. The double-joint final PCR products were used to transform A. brassicicola protoplasts as described by [53]. The A. brassicicola wild-type Abra43 was used to obtain the hygromycin-resistant strains deficient for each GST. Potential transformants were prescreened by PCR with relevant primer combinations (Additional file 2) to confirm integration of the replacement cassette at the targeted locus. Two putative gene replacement mutants for each construct were further purified by three rounds of single-spore isolation and then confirmed by PCR.

\section{Infection assays}

For plant infection assays on Brassica oleracea plants (var. Bartolo), $5 \mu \mathrm{L}$ drops of $A$. brassicicola conidia suspension $\left(10^{5}, 10^{4}\right.$ or $10^{3}$ conidia/mL in sterile water) were inoculated on leaves from 5 week-old plants. Inocula were symmetrically deposited on the left and right sides from the central vein. The plants were then maintained under saturating humidity (100\% relative humidity). Symptoms were observed and samples collected at 2, 4, 6 days postinoculation (dpi) for $A b G s t$ expression analyses.

\section{Expression and purification of the recombinant proteins}

GST coding sequences were amplified from the cDNAs obtained as described above, using the Phusion ${ }^{\text {th }}$ Hot Start High Fidelity DNA polymerase (Finnzymes) and relevant primer sets (Additional file 2). The PCR products were cloned into the $\mathrm{NcoI}$ and either BamHI sites (AbGTT1.2, AbGSTO1, AbMAPEG1 and AbGSTFuA1) or XhoI (AbUre2pB1) of the pET-14b vector (Novagen) resulting in a construction devoid of an His-Tag. The recombinant plasmids were then used to transform the Escherichia coli strain BL21 (DE3) co-transformed by the chloramphenicol-resistant plasmid (pRARE) in order to provide the rare t-RNAs for AUA, AGG, AGA, CUA,
CCC, and GGA. Cultures were progressively amplified up to $2 \mathrm{~L}$ in $\mathrm{LB}$ medium supplemented with ampicillin and kanamycin at $37{ }^{\circ} \mathrm{C}$. Protein expression was induced at the exponential phase by adding $100 \mu \mathrm{M}$ isopropyl $\beta$ D-thiogalactopyranoside (ITPG) for $4 \mathrm{~h}$ at $37^{\circ} \mathrm{C}$. The cultures were then centrifuged for $15 \mathrm{~min}$ at $4400 \times \mathrm{g}$. The pellets were suspended in $30 \mathrm{~mL}$ of $\mathrm{TE} \mathrm{NaCl}$ (30 mM Tris- $\mathrm{HCl}, \mathrm{pH}$ 8.0, $1 \mathrm{mM}$ EDTA, $200 \mathrm{mM} \mathrm{NaCl}$ ) buffer. Cell lysis was performed on ice by sonication $(2 \times 2 \mathrm{~min}$ at $1 \mathrm{~min}$ intervals), and the soluble and insoluble fractions were separated by centrifugation for $30 \mathrm{~min}$ at $27,000 \times g$ at $4{ }^{\circ} \mathrm{C}$. The soluble fraction (supernatant) was then fractionated with ammonium sulfate in two steps, and the protein fraction precipitating between 40 and $80 \%$ of the saturation contained the recombinant protein, as estimated by $15 \%$ SDS-PAGE. The protein was purified by size exclusion chromatography after loading on an ACA44 $(5 \times 75 \mathrm{~cm})$ column equilibrated in $\mathrm{TE} \mathrm{NaCl}$ buffer. Fractions containing the protein were pooled, dialyzed by ultrafiltration to remove $\mathrm{NaCl}$, and loaded onto a DEAE cellulose column (Sigma) in TE (30 mM Tris-HCl, pH 8.0, 1 mM EDTA) buffer. The proteins were eluted using a $0-0.4 \mathrm{M} \mathrm{NaCl}$ gradient. Finally, the fractions of interest were pooled, dialyzed, concentrated by ultrafiltration under nitrogen pressure (YM10 membrane; Amicon). Purity was checked by SDS-PAGE. Protein concentrations were determined spectrophotometrically using the specific molar extinction coefficient at $280 \mathrm{~nm}$ of each GST as calculated online (http://web.expasy.org/prot param/) using the ProtParam tool: $42400 \mathrm{~cm}^{-1} \mathrm{M}^{-1}, 46$ $410 \mathrm{~cm}^{-1} \mathrm{M}^{-1}, 60390 \mathrm{~cm}^{-1} \mathrm{M}^{-1}, 49390 \mathrm{~cm}^{-1} \mathrm{M}^{-1}$ for AbGTT1.2, AbGSTO1, AbUre2pB1 and AbGSTFuA1, respectively.

\section{Activity measurement}

GSH transferase activity was spectrophotometrically assessed with Al-ITC, Bz-ITC and Ph-ITC prepared in methanol and 1-chloro-2,4-dinitrobenzene (CDNB) prepared in DMSO. The increased absorbance arising from the formation of the S-glutathionylated adduct was monitored at $274 \mathrm{~nm}$ for ITC and $340 \mathrm{~nm}$ for CDNB. Reactions with CDNB were performed in $100 \mathrm{mM}$ phosphate buffer ( $\mathrm{pH} 7.5)$, in the presence of GSH $(5 \mathrm{mM})$ while the reaction with ITC was performed at $\mathrm{pH} 6.5$ with an identical GSH concentration. Peroxidase activities were monitored as follows: $1 \mathrm{mM}$ peroxide (hydrogen peroxide, and cumene hydroperoxide) in $30 \mathrm{mM}$ Tris$\mathrm{HCl}(\mathrm{pH} \mathrm{8.0)}$, was incubated in the presence of $2 \mathrm{mM}$ GSH, $200 \mu \mathrm{M}$ NADPH, 0.5 IU glutathione reductase. The activity was monitored according to the decrease in absorbance at $340 \mathrm{~nm}$ arising from NADPH oxidation in this coupled enzyme assay system. Hydroxyethyldisulfide (HED) was incubated with GSH to allow the spontaneous formation of glutathionylated $\beta$-mercaptoethanol ( $\beta$-ME- 
SG). Then, the $\beta$-ME-SG deglutathionylation test was monitored using the coupled system but by using $1 \mathrm{mM}$ HED instead of the peroxides. The reactions were started by adding the purified enzyme $(0.1 \mu \mathrm{M})$ and monitored with a Cary 50 UV-Visible spectrophotometer (VARIAN). Catalytic parameters were calculated using the $\mathrm{GraphPad}^{\circ}$ software.

\section{Sequence analysis}

Amino-acid sequence alignments were done by CLUSTALW and the tree was constructed with the neighbor joining method in MEGA 5.0 software [54]. Hydropathy curves were generated according to Kyte and Doolitle [55].

\section{Additional files}

Additional file 1: Structural features of AbMAPEG1. (A) Protein sequence of AB08663.1. Amino-acids in red are conserved in the specific pattern characteristic for MAPEG members belonging to the MGST3 subfamily [45]. Underlined residues are conserved in all members of the MAPEG family [46]. (B) Hydrophobicity plots according to [55] of the protein sequences from AB08663.1 (left) and human MGST3 (Swissprot identifier 014880) (right).

Additional file 2: List of primers.

Additional file 3: List of sequences used to generate the phylogenetic tree of GSTs.

\section{Abbreviations \\ B-ME-SG: glutathionylated $\beta$-mercaptoethanol; CDNB: 1-chloro-2, 4-dinitrobenzene; $\mathrm{Cu}-\mathrm{OOH}$ : Cumene peroxide; GSH: Glutathione; GST: Glutathione-S-transferase; HED: Hydroxyethyldisulfide; ITC: Isothiocyanate.}

\section{Competing interests}

The authors declare that they have no competing interests.

\section{Authors' contributions}

BC carried out the molecular genetic and mutant phenotyping studies. NBS produced the recombinant GSTs. MMR performed the phylogenetic analysis. MMR, BC and EG performed the purification of recombinant GSTs and activity measurements. PS and TG conceived the study. All authors participated in the design of the experiments as well as analysis of the results. BC drafted the initial manuscript and all authors participated in the editing and approved its final version.

\section{Acknowledgments}

We wish to thank Anne Thullier, Tiphaine Dhalleine, Sandrine Pigné and Bruck Taddese for technical assistance and David Manley for editing the manuscript. We are grateful to members of the INEM and COMIC facilities for providing plants and preserving fungal strains, respectively. The UMR1136 is supported by a grant overseen by the French National Research Agency (ANR) as part of the "Investissements d'Avenir" program (ANR-11-LABX-0002-01, Lab of Excellence ARBRE).

\section{Author details}

'Université d'Angers, UMR 1345 IRHS, SFR 4207 QUASAV, 2 Bd Lavoisier, Angers cedex F-49045, France. ${ }^{2}$ INRA, UMR 1345 IRHS, 42 rue Georges Morel, Beaucouzé Cedex F-49071, France. ${ }^{3}$ Agrocampus-Ouest, UMR 1345 IRHS, 2 rue le Nôtre, Angers cedex F-49045, France. ${ }^{4}$ Université de Lorraine, UMR1 136 Interactions Arbres-Microorganismes, Vandoeuvre-lès, F-54500 Nancy, France. ${ }^{5}$ INRA, UMR1136 Interactions Arbres-Microorganismes, F-54280 Champenoux, France.

Received: 2 April 2015 Accepted: 3 June 2015

Published online: 18 June 2015

\section{References}

1. Thomma B, Nelissen I, Eggermont K, Broekaert W. Deficiency in phytoalexin production causes enhanced susceptibility of Arabidopsis thaliana to the fungus Alternaria brassicicola. Plant J. 1999;19:163-71.

2. Tierens K, Thomma B, Brouwer M, Schmidt J, Kistner K, Porzel A, et al. Study of the role of antimicrobial glucosinolate-derived isothiocyanates in resistance of Arabidopsis to microbial pathogens. Plant Physiol. 2001;125:1688-99.

3. Fahey JW, Zalcmann AT, Talalay P. The chemical diversity and distribution of glucosinolates and isothiocyanates among plants. Phytochemistry. 2001;56:5-51.

4. Fenwick GR, Heaney RK, Mullin WJ. Glucosinolates and their breakdown products in cruciferous crops, foods and feedingstuffs. Food Chem. 1983;11:249-71.

5. Sellam A, Dongo A, Guillemette T, Hudhomme P, Simoneau P. Transcriptional responses to exposure to the brassicaceous defence metabolites camalexin and allyl-isothiocyanate in the necrotrophic fungus Alternaria brassicicola. Mol Plant Pathol. 2007:8:195-208.

6. Hayes JD, Flanagan JU, Jowsey IR. Glutathione transferases. Annu Rev Pharmacol Toxicol. 2005;45:51-88.

7. Kolm RH, Danielson UH, Zhang Y, Talalay P, Mannervik B. Isothiocyanates as substrates for human glutathione transferases: structure-activity studies. Biochem J. 1995;311:453-9.

8. Bednarek P, Pislewska-Bednarek M, Svatos A, Schneider B, Doubsky J, Mandsurova $\mathrm{M}$, et al. A glucosinolate metabolism pathway in living plant cells mediates broadspectrum antifungal defense. Science. 2009:323:101-6.

9. Mathieu Y, Prosper P, Favier F, Harvengt L, Didierjean C, Jacquot JP, et al. Diversification of fungal specific class A glutathione transferases in saprotrophic fungi. PLoS One. 2013;8, e80298.

10. Francis F, Vanhaelen N, Haubruge E. Glutathione S-transferases in the adaptation to plant secondary metabolites in the Myzus persicae aphid. Arch Insect Biochem Physiol. 2005;58:166-74.

11. Hasegawa K, Miwa S, Tsutsumiuchi K, Miwa J. Allyl isothiocyanate that induces GST and UGT expression confers oxidative stress resistance on C. elegans, as demonstrated by nematode biosensor. PLoS One. 2010;5, e9267.

12. Rahmanpour S, Backhouse D, Nonhebel HM. Induced tolerance of Sclerotinia sclerotiorum to isothiocyanates and toxic volatiles from Brassica species. Plant Pathol. 2009;58:479-86.

13. Oakley AJ, Lo Bello M, Nuccetelli M, Mazzetti AP, Parker MW. The ligandin (non-substrate) binding site of human Pi class glutathione transferase is located in the electrophile binding site (H-site). J Mol Biol. 1999;291:913-26.

14. Dixon DP, Lapthorn A, Madesis P, Mudd EA, Day A, Edwards R. Binding and glutathione conjugation of porphyrinogens by plant glutathione transferases. J Biol Chem. 2008;283:20268-76.

15. Bilang J, Macdonald H, King PJ, Sturm A. A soluble auxin-binding protein from Hyoscyamus muticus is a glutathione-S-transferase. Plant Physiol. 1993;102:29-34.

16. Conn S, Curtin C, Bezier A, Franco C, Zhang W. Purification, molecular cloning, and characterization of glutathione Stransferases (GSTs) from pigmented Vitis vinifera L. cell suspension cultures as putative anthocyanin transport proteins. J Exp Bot. 2008:59:3621-34.

17. Mathieu Y, Prosper P, Buee M, Dumarcay S, Favier F, Gelhaye E, et al, Characterization of a Phanerochaete chrysosporium glutathione transferase reveals a novel structural and functional class with ligandin properties. J Biol Chem. 2012;287:39001-11.

18. Awasthi YC, Yang Y, Tiwari NK, Patrick B, Sharma A, Li J, et al. Regulation of 4-hydroxynonenal-mediated signaling by glutathione S-transferases.". Free Radic Biol Med. 2004:37:607-19.

19. Bai M, Zhou JM, Perrett $\mathrm{S}$. The yeast prion protein Ure2 shows glutathione peroxidase activity in both native and fibrillar forms. J Biol Chem. 2004;279:50025-30.

20. Burns C, Geraghty R, Neville C, Murphy A, Kavanagh K, Doyle S. Identification, cloning, and functional expression of three glutathione transferase genes from Aspergillus fumigatus. Fung Genet Biol. 2005;42:319-27.

21. Choi J, Lou W, Vancura A. A novel membrane-bound glutathione S-transferase functions in the stationary phase of the yeast Saccharomyces cerevisiae. J Biol Chem. 1998;273:29915-22

22. Eaton DL, Bammler TK. Concise review of the glutathione S-transferases and their significance to toxicology. Toxicol Sci. 1999;49:156-64.

23. Joubert A, Bataille-Simoneau N, Campion C, Guillemette T, Hudhomme P, lacomi-Vasilescu B, et al. Cell wall integrity and high osmolarity glycerol pathways are required for adaptation of Alternaria brassicicola to cell wall 
stress caused by brassicaceous indolic phytoalexins. Cell Microbiol. 2011;13:62-80.

24. Joubert A, Simoneau P, Campion C, Bataille-Simoneau N, lacomi-Vasilescu B, Poupard $\mathrm{P}$, et al. Impact of unfolded protein response on the pathogenicity of the necrotrophic fungus Alternaria brassicicola. Mol Microbiol. 2011;79:1305-24

25. Calmes B, Guillemette T, Teyssier L, Siegler B, Pigné S, Landreau A, et al. Role of mannitol metabolism in the pathogenicity of the necrotrophic fungus Alternaria brassicicola. Front Plant Sci. 2013;4:131.

26. Sellam A, Poupard P, Simoneau P. Molecular cloning of AbGst1 encoding a glutathione transferase differentially expressed during exposure of Alternaria brassicicola to isothiocyanates. FEMS Microbiol Lett. 2006;258:241-9.

27. McGoldrick S, O'Sullivan S, Sheehan D. Glutathione transferase-like proteins encoded in genomes of yeasts and fungi: insights into evolution of a multifunctional protein superfamily. FEMS Microbiol Lett. 2005;242:1-12.

28. Morel M, Ngadin AA, Droux M, Jacquot JP, Gelhaye E. The fungal glutathione $S$ transferase system. Evidence of new classes in the wooddegrading basidiomycete Phanerochaete chrysosporium. Cell. Mol. Life Sci. 2009;66:3711-25.

29. Thuillier A, Ngadin AA, Thion C, Billard P, Jacquot JP, Gelhaye E, et al. Functional diversification of fungal glutathione transferases from the ure2p class. Int J Evol Biol. 2011;938308.

30. Morel M, Meux E, Mathieu Y, Thuillier A, Chibani K, Harvengt L, et al. Xenomic networks variability and adaptation traits in wood decaying fungi.". Microb Biotechnol. 2013;6:248-63.

31. Garcera A, Barreto L, Piedrafita L, Tamarit J, Herrero E. Saccharomyces cerevisiae cells have three Omega class glutathione S-transferases acting as 1-Cys thiol transferases. Biochem J. 2006;398:187-96.

32. Todorova $T$, Petrova VY, Vuilleumier $S$, Kujumdzieva AV. Response to different oxidants of Saccharomyces cerevisiae ure $2 \Delta$ mutant. Arch Microbiol. 2009;191:837-45.

33. Thuillier A, Roret T, Favier F, Gelhaye E, Jacquot JP, Didierjean C, et al. Atypical features of a Ure2p glutathione transferase from Phanerochaete Chrysosporium. FEBS Lett. 2013;587:2125-30.

34. Wadington MC, Ladner JE, Stourman NV, Harp JM, Armstrong RN. Analysis of the structure and function of YfcG from Escherichia coli reveals an efficient and unique disulfide bond reductase. Biochemistry. 2009;48:6559-61.

35. Zhang Y. The molecular basis that unifies the metabolism, cellular uptake and chemopreventive activities of dietary isothiocyanates. Carcinogenesis. 2012;2-9.

36. Zhang Y. Role of glutathione in the accumulation of anticarcinogenic isothiocyanates and their glutathione conjugates by murine hepatoma cells. Carcinogenesis. 2000;21:1175-82.

37. Zhang Y. Molecular mechanism of rapid cellular accumulation of anticarcinogenic isothiocyanates. Carcinogenesis. 2001;22:425-31.

38. Herrero E, Ros J, Tamarit J, Belli G. Glutaredoxins in fungi. Photosynth Res. 2006:89:127-40.

39. Mariani D, Mathias CJ, da Silva CG, Herdeiro RS, Pereira R, Panek AD, et al. Involvement of glutathione transferases, Gtt1 and Gtt2, with oxidative stress response generated by $\mathrm{H} 2 \mathrm{O} 2$ during growth of Saccharomyces cerevisiae. Redox Rep. 2006;13:246-54.

40. Board PG. The omega-class glutathione transferases: structure, function, and genetics. Drug Metab Rev. 2011;43:226e235.

41. Whitbread AK, Masoumi A, Tetlow N, Schmuck E, Coggan M, Board PG. Characterization of the omega class of glutathione transferases. Methods Enzymol. 2005;401:78-99.

42. Geng F, Tang L, Li Y, Yang L, Choi KS, Kazim AL, et al. Allyl isothiocyanate arrests cancer cells in mitosis, and mitotic arrest in turn leads to apoptosis via $\mathrm{BCL}-2$ phosphorylation. J Biol Chem. 2011;286:32259-67.

43. Youn HS, Kim YS, Park ZY, Kim SY, Choi NY, Joung SM, et al. Sulforaphane suppresses oligomerization of TLR4 in a thiol-dependent manner. J Immunol. 2010;184:411-9.

44. Goosen TC, Kent UM, Brand L, Hollenberg PF. Inactivation of cytochrome P450 2B1 by benzyl isothiocyanate, a chemopreventative agent from cruciferous vegetables. Chem Res Toxicol. 2000;13:1349-59.

45. Bresell $A$, Weinander $R$, Lundqvist $G$, Raza $H$, Shimoji M, Sun $T H$, et al. Bioinformatic and and enzymatic characterization of the MAPEG superfamily. Febs J. 2005;272:1688-703.

46. Jakobsson PJ, Mancini JA, Riendeau D, Ford-Hutchinson AW. Identification and characterization of a novel microsomal enzyme with glutathione- dependent transferase and peroxidase activities. J Biol Chem. 1997:272:22934-9.

47. Thuillier A, Chibani K, Belli G, Herrero E, Dumarcay S, Gérardin P, et al. Transcriptomic responses of Phanerochaete chrysosporium to oak acetonic extracts : focus on a new glutathione transferase. Appl Environ Microbiol. 2014;80:6316-27.

48. Prins T, Wagemakers L, Schouten A, van Kan J. Cloning and characterization of a glutathione S-transferase homologue from the plant pathogenic fungus Botrytis cinerea. Mol Plant Pathol. 2000;1:169-78.

49. Joubert A, Calmes B, Berruyer R, Pihet M, Bouchara JP, Simoneau P, et al. Laser nephelometry applied in an automated microplate system to study filamentous fungus growth. Biotechniques. 2010;48:399-404.

50. Winer J, Jung CK, Shackel I, Williams PM. Development and validation of real-time quantitative reverse transcriptase-polymerase chain reaction for monitoring gene expression in cardiac myocytes in vitro. Anal Biochem. $1999 ; 270: 41-9$

51. Yu JH, Hamari Z, Han KH, Seo JA, Reyes-Dominguez Y, Scazzocchio C. Double-joint PCR: a PCR-based molecular tool for gene manipulations in filamentous fungi. Fungal Genet Biol. 2004;41:973-81.

52. Sweigard JA, Carroll AM, Kang S, Farrall L, Chumley FG, Valent B. Identification, cloning, and characterization of PWL2, a gene for host species specificity in the rice blast fungus. Plant Cell. 1995;7:1221-33.

53. Cho Y, Davis JW, Kim KH, Wang J, Sun QH, Cramer Jr RA, et al. A high throughput targeted gene disruption method for Alternaria brassicicola functional genomics using linear minimal element (LME) constructs. Mol Plant Microbe Interact. 2006;19:7-15

54. Tamura K, Peterson D, Peterson N, Stecher G, Nei M, Kumar S. MEGA5: molecular evolutionary genetics analysis using maximum likelihood evolutionary distance, and maximum parsimony methods. Mol Biol Evol. 2011;28:2731-9.

55. Kyte J, Doolittle RF. A simple method for displaying the hydropathic character of a protein. J Mol Biol. 1982;157:105-32.

\section{Submit your next manuscript to BioMed Central and take full advantage of:}

- Convenient online submission

- Thorough peer review

- No space constraints or color figure charges

- Immediate publication on acceptance

- Inclusion in PubMed, CAS, Scopus and Google Scholar

- Research which is freely available for redistribution 